\title{
Tarifpolitischer Halbjahresbericht: Eine Zwischenbilanz der Lohn- und Gehaltsrunde 2012
}

\author{
Die Tarifrunde 2012 hat lohn- und verteilungspolitisch erfolgreich begonnen. Die Ab- \\ schlussraten haben sich im Vergleich zum Vorjahr deutlich erhöht, der kostenneutrale \\ Verteilungsspielraum wurde bislang ausgeschöpft. Voraussichtlich werden sich die realen \\ Tarifeinkommen angesichts einer sehr moderaten Preisentwicklung im Schnitt spürbar \\ erhöhen. Zudem gelang es den Gewerkschaften, in einigen qualitativen Fragen, wie bei \\ der Übernahme der Auszubildenden und der Regulierung der Leiharbeit, tarifpolitische \\ Fortschritte durchzusetzen.
}

REINHARD BISPINCK, WSI-TARIFARCHIV

\section{1. Übersicht}

Die wirtschaftliche Entwicklung hatte sich bereits vor Beginn der Tarifrunde eingetrübt. Nach zunächst starkem Wachstum kam es zum Jahresende 2011 infolge nachlassender Auslandsnachfrage sogar zu einem leichten Produktionsrückgang (IMK 2012). Auch die Perspektiven für 2012 wurden in den Prognosen der Wirtschaftsinstitute zurückhaltender beurteilt. Gleichwohl gab es für die Tarifforderungen der Gewerkschaften - ähnlich wie im Vorjahr - breite öffentliche Unterstützung. Zum einen setzte sich die Erkenntnis durch, dass eine Stabilisierung der wirtschaftlichen Entwicklung in Deutschland einer kräftigeren Binnennachfrage bedarf. Zum anderen war auch die anhaltende öffentliche Debatte über faire Löhne hilfreich, die einmal mehr durch die exorbitanten und erneut stark gestiegenen Management-Gehälter, insbesondere in den DAX-Unternehmen, gespeist wurde (Towers-Watson 2012). Trotz dieser Unterstützung verliefen die Tarifverhandlungen nicht konfliktfrei. Im öffentlichen Dienst und in der Metallindustrie kam es zu umfangreichen Warnstreiks, an denen sich jeweils mehrere hunderttausend Beschäftigte beteiligten.

Die Lohn- und Gehaltsforderungen der Gewerkschaften bewegten sich in der diesjährigen Tarifrunde überwiegend zwischen 6 und 7 \%. Die Gewerkschaft Nahrung-GenussGaststätten (NGG) forderte für ihre Branchen zwischen 5 und $6 \%$ sowie tarifliche Mindestentgelte von $8,50 € .6 \%$ forderten die Gewerkschaften auch in den Branchen chemische Industrie, Bankgewerbe sowie in der Landwirtschaft (hier: 5,9 \%). 6,5 \% betrug die Forderung in der Metall- und Elektroindustrie sowie bei Volkswagen und bei der Deutschen Telekom AG. Im öffentlichen Dienst (Bund und Gemeinden) forderte die Vereinte Dienstleistungsgewerkschaft (ver.di) ebenfalls 6,5\%, jedoch mindestens $200 €$. Das bedeutete für die unteren Entgeltgruppen Erhöhungen um bis zu $10 \%$ und mehr. $7 \%$ Tariferhöhung forderte ver.di bei der Deutschen Post und im privaten Verkehrsgewerbe Baden-Württemberg.

Neben den reinen Entgeltforderungen spielten in einigen Tarifbereichen auch qualitative Tarifforderungen eine wichtige Rolle: In der Metallindustrie forderte die IG Metall Regelungen zur unbefristeten Übernahme der Ausgebildeten sowie eine Begrenzung und stärkere Mitbestimmungsrechte bei der Leiharbeit. Im Bankgewerbe forderte ver.di u. a. Regelungen zum Gesundheits- und Belastungsschutz. Und in der chemischen Industrie stand die Weiterentwicklung des Tarifvertrages „Lebensarbeitszeit und Demografie“ auf der tarifpolitischen Tagesordnung.

Der Kündigungsterminkalender gab folgenden zeitlichen Ablauf der Tarifrunde vor:

- Ende Dezember 2011 liefen die Tarifverträge für die Deutsche Post AG und bei den Ortskrankenkassen aus; - Ende Januar 2012 folgte die Deutsche Telekom AG; 
- im Februar 2012 endete die Laufzeit der Verträge im öffentlichen Dienst (Bund und Gemeinden) sowie im Bankgewerbe;

- Ende März folgten die Metall- und Elektroindustrie sowie einige Bereiche des privaten Verkehrsgewerbes;

- Ende April standen Verträge in verschiedenen Regionen des Kfz-Gewerbes sowie des Hotel- und Gaststättengewerbes zur Verhandlung an;

- Ende Mai folgte die Volkswagen AG. Ebenfalls Ende Mai sowie Ende Juni liefen die Verträge in der chemischen Industrie aus.

Wegen der lang laufenden Abschlüsse aus den Vorjahren gab es in zahlreichen Branchen in diesem Jahr keine Lohnrunde. Dazu zählen u. a. der Einzel- und Großhandel, das Bauhauptgewerbe, die Druckindustrie, das Versicherungsgewerbe sowie der öffentliche Dienst im Bereich der Länder. Ein Blick auf die Tarifabschlüsse zeigt für das erste Halbjahr folgendes Bild (vgl. auch Übersicht 1):

- März: Den ersten großen Abschluss legte der öffentliche Dienst, Bund Gemeinden am 31.3. vor: Er kam nach umfangreichen bundesweiten Warnstreiks zustande und sieht eine Tarifanhebung von 3,5 \% ab 1.3.2012 vor sowie zwei Stufenanhebungen von jeweils $1,4 \%$ ab 1.1.2013 und 1.8.2013. Die Gesamtlaufzeit beträgt 24 Monate (siehe Abschnitt 3.1).

- April: Auch bei der Deutschen Telekom AG hat der Abschluss vom 28.4.2012 eine Laufzeit von zwei Jahren: Nach drei Nullmonaten folgen zum 1.5.2012 eine Tariferhöhung um 2,3\% sowie zwei Stufenerhöhungen von jeweils 2,1\% ab 1.1.2013 und 1.9.2013. Für die T-Servicegesellschaften wurde ein gesonderter Abschluss vereinbart.

- Mai: Im Hotel- und Gaststättengewerbe Nordrhein-Westfalen erreichte die NGG am 4.5.2012 nicht nur eine Tariferhöhung in drei Stufen um 3,1 \% ab 1.6.2012 sowie jeweils $1,6 \%$ ab 1.2.2013 und 1.9.2013, sondern auch eine strukturelle Anpassung der untersten Entgeltgruppe mit einer Anhebung auf 8,50€ spätestens ab 1.9.2013.

In der Metall- und Elektroindustrie vereinbarte die IG Metall nach umfangreichen Warnstreiks im Pilotabschluss in Baden-Württemberg am 19.5.2012 nach einem Nullmonat eine Tarifanhebung von 4,3\% bei einer Laufzeit von insgesamt 13 Monaten. Außerdem wurden die unbefristete Übernahme der Ausgebildeten als Grundsatz festgeschrieben und die Mitspracherechte des Betriebsrates bei Leiharbeit verbessert. Am 22.5.2012 einigte sich die IG Metall mit den beiden Zeitarbeitsverbänden Bundesarbeitgeberverband der Personaldienstleister (BAP) und Interessenverband Deutscher Zeitarbeitsunternehmen (iGZ) auf tarifliche Branchenzuschläge von 15 bis $50 \%$, gestaffelt nach ununterbrochener Beschäftigungsdauer im Kundenbetrieb. Der Tarifabschluss bei Volkswagen vom 31.5.2012 sah ein ähnliches Volumen (ohne Nullmonat) vor (siehe Abschnitt 3.2).
In der chemischen Industrie sieht der Abschluss vom 24.5.2012 nach einem Nullmonat eine tarifliche Entgeltsteigerung von 4,5\% für 18 Monate vor. Vereinbart wurden außerdem eine Aufstockung der betrieblichen Demografiefonds sowie Regelungen zur Arbeitszeitgestaltung insbesondere für ältere Beschäftigte (siehe Abschnitt 3.3).

- Juni: Für das Bankgewerbe konnte ver.di eine Tarifanhebung von 2,9 \% ab 1.7.2012, eine Stufenerhöhung von 2,5 \% ab 1.7.2013 sowie eine Pauschalzahlung von $350 €$ für März bis Juni erreichen. Die Gesamtlaufzeit beträgt 26 Monate. Vereinbart wurde ferner eine Erklärung zum Gesundheitsschutz der Beschäftigten.

\section{Ergebnisse im gesamtwirtschaftlichen Überblick}

Im 1. Halbjahr des Jahres 2012 schlossen die Gewerkschaften des DGB Einkommenstarifverträge für rund 7,1 Mio. Beschäftigte $\mathrm{ab}$, darunter rund 0,9 Mio. in den neuen Bundesländern. Das entspricht etwa 38 \% der von Tarifverträgen erfassten Arbeitnehmerinnen und Arbeitnehmer.

Die durchschnittliche Abschlussrate einschließlich aller Stufenanhebungen, die während der Laufzeit der Verträge wirksam werden, beträgt 4,8 \% (West: 4,8\%, Ost: 5,0 \%). Berücksichtigt werden bei der Abschlussrate nur die tabellenwirksamen Tarifanhebungen, während Pauschal- und zusätzliche Einmalzahlungen außen vor bleiben.

Berücksichtigt man ausschließlich die im Jahr 2012 in Kraft tretenden Tariferhöhungen, ergibt sich eine $\mathrm{Ab}$ schlussrate von 3,9 \% (West: 4,0 \%, Ost: 3,8 \%). Damit liegt die Abschlussrate, die 2012 wirksam wird, um einen Prozentpunkt höher als im Vorjahr (2011:2,9 \%). Differenziert man diese Größe nach Wirtschaftsbereichen, dann ergibt sich in diesem Jahr folgende Streuung: Am unteren Ende liegt der Bereich Private Dienstleistungen, Organisationen ohne Erwerbszweck sowie Kreditinstitute und Versicherungsgewerbe mit je 2,9\%. Es folgt der Bereich Energieund Wasserversorgung, Bergbau mit 3,1\%. Die Bereiche Verbrauchsgütergewerbe, Nahrungs- und Genussmittelgewerbe sowie das Baugewerbe weisen 3,4\% aus. Die Bereiche Verkehr und Nachrichtenübermittlung sowie Gebietskörperschaften, Sozialversicherung erreichen 3,5\%. Deutlich über dem Durchschnitt liegen das Investitionsgütergewerbe sowie das Grundstoff- und Produktionsgütergewerbe mit 4,3 bzw. 4,4\%.

Für rund $70 \%$ der Beschäftigten mit Neuabschlüssen traten die Tarifsteigerungen mit zeitlicher Verzögerung in Kraft. Überwiegend war es lediglich ein Verzögerungsmonat. Da ist es nachvollziehbar, dass lediglich knapp $10 \%$ der betroffenen Beschäftigten für diese Nullmonate Pauschalzahlungen von durchschnittlich $91 €$ pro Verzögerungsmonat erhielten. Die Laufzeit der neu abgeschlossenen Tarifverträ- 
Ausgewählte Tarifforderungen und -abschlüsse in der Tarifrunde 2012

\begin{tabular}{|c|c|c|c|c|}
\hline Abschlussdatum & Tarifbereich & Forderung & Abschluss & Weitere Regelungen \\
\hline 12.01. & Deutsche Post AG & $7,0 \%$ & $\begin{array}{l}400 € \text { Pauschale für } 3 \text { Monate } \\
4,0 \% \text { ab } 01.04 .12 \\
\text { LZ: } 15 \text { Monate bis } 31.03 .13\end{array}$ & \\
\hline 31.03. & $\begin{array}{l}\text { Öffentlicher Dienst } \\
\text { Bund und Gemeinden }\end{array}$ & $\begin{array}{l}6,5 \% \\
\text { mind. } 200 €\end{array}$ & $\begin{array}{l}\text { 3,5 \% ab 01.03.12 } \\
\text { 1,4 \% Stufenerhöhung ab 01.01.13 } \\
\text { 1,4 \% Stufenerhöhung ab 01.08.13 } \\
\text { LZ: } 24 \text { Monate bis } 28.02 .14\end{array}$ & $\begin{array}{l}\text { Übernahmeregelung Ausgebildeter } \\
\text { Sonderzahlung für AN an Flughäfen }\end{array}$ \\
\hline 28.04. & Deutsche Telekom AG & $6,5 \%$ & $\begin{array}{l}3 \text { Nullmonate } \\
2,3 \% \text { ab } 01.05 .12 \\
2,1 \% \text { Stufenerhöhung ab } 01.01 .13 \\
\text { 2,1 \% Stufenerhöhung ab } 01.08 .13 \\
\text { LZ: } 24 \text { Monate bis } 31.01 .14\end{array}$ & $\begin{array}{l}\text { Gesonderter Abschluss für Deutsche } \\
\text { Telekom Servicegesellschaften }\end{array}$ \\
\hline 04.05. & Hotels und Gaststätten NRW & $6,0 \%$ & $\begin{array}{l}1 \text { Nullmonat } \\
3,1 \% \text { ab } 01.06 .12 \\
1,6 \% \text { Stufenerhöhung ab } 01.02 .13 \\
\text { 1,6 \% Stufenerhöhung ab } 01.09 .13 \\
\text { LZ: } 24 \text { Monate bis } 30.04 .14\end{array}$ & $\begin{array}{l}\text { Strukturelle Anpassung der unteren } \\
\text { Entgeltgruppe, Anhebung auf } 8,50 €\end{array}$ \\
\hline 08.05. & $\begin{array}{l}\text { Privates Verkehrsgewerbe } \\
\text { Baden-Württemberg } \\
\text { (ohne Südbaden) }\end{array}$ & $7,0 \%$ & $\begin{array}{l}\text { 3,5 \% ab 01.04.12 } \\
\text { 2,5\% Stufenerhöhung ab 01.04.13 } \\
\text { LZ: } 24 \text { Monate bis 31.03.14 }\end{array}$ & \\
\hline $\begin{array}{l}\text { 19.05. / } \\
22.05 .\end{array}$ & $\begin{array}{l}\text { Metall- und } \\
\text { Elektroindustrie }\end{array}$ & $6,5 \%$ & $\begin{array}{l}1 \text { Nullmonat } \\
4,3 \% \text { ab } 01.05 .12 \\
\text { LZ: } 13 \text { Monate bis } 30.04 .13\end{array}$ & $\begin{array}{l}\text { Unbefristete Übernahme Ausgebildeter } \\
\text { als Grundsatz, Mitbestimmung und } \\
\text { Branchenzuschläge bei Leiharbeit }\end{array}$ \\
\hline 24.05. & $\begin{array}{l}\text { Chemische } \\
\text { Industrie }\end{array}$ & $6,0 \%$ & $\begin{array}{l}\text { 1 Nullmonat } \\
\text { 4,5 \% für } 18 \text { Monate } \\
\text { LZ: } 19 \text { Monate reg. unterschiedlich bis } \\
\text { 31.12.13, 31.01. bzw. } 28.02 .14\end{array}$ & $\begin{array}{l}\text { Aufstockung der Demografiefonds, } \\
\text { Arbeitszeitgestaltung u. a. für Ältere }\end{array}$ \\
\hline 30.05. & $\begin{array}{l}\text { Kfz-Gewerbe } \\
\text { Baden-Württemberg }\end{array}$ & $6,5 \%$ & $\begin{array}{l}1 \text { Nullmonat } \\
\text { 4,0 \% ab } 01.06 .12 \\
\text { LZ: } 13 \text { Monate bis } 31.05 .13\end{array}$ & $\begin{array}{l}\text { Regelung zur 12-monatigen Übernahme } \\
\text { Ausgebildeter }\end{array}$ \\
\hline 31.05. & Volkswagen AG & $6,5 \%$ & $\begin{array}{l}\text { 4,3 \% ab } 01.06 .12 \\
\text { LZ: } 13 \text { Monate bis } 30.06 .13\end{array}$ & Regelungen zur Leiharbeit \\
\hline 06.06. & Bankgewerbe & $6,0 \%$ & $\begin{array}{l}350 € \text { Pauschale für } 4 \text { Monate } \\
2,9 \% \text { ab } 01.07 .12 \\
\text { 2,5\% Stufenerhöhung ab } 01.07 .13 \\
\text { LZ: } 26 \text { Monate bis } 30.04 .14\end{array}$ & $\begin{array}{l}\text { Verlängerung Vorruhestand, Erklärung zur } \\
\text { Ausbildung, zum Gesundheits- und } \\
\text { Anlegerschutz }\end{array}$ \\
\hline
\end{tabular}

ge beträgt im Durchschnitt 17,1 Monate, damit wurde der Trend zu länger laufenden Lohn- und Gehaltstarifverträgen in diesem Jahr gebrochen. Das ist maßgeblich auf den Tarifvertrag für die Metallindustrie mit einer Laufzeit von lediglich 13 Monaten zurückzuführen. Längere Laufzeiten ergaben sich mit 26 Monaten im Bankgewerbe sowie mit 24 Monaten im Bereich Gebietskörperschaften, Sozialversicherung.

Die Aussagekraft der tariflichen Abschlussrate ist begrenzt. Generell gilt: Aussagen zur Veränderung von wirt- schaftlichen Kennziffern müssen auf einen festen Zeitraum normiert werden, um vergleichbar zu sein. Veränderungsraten beziehen sich in der Regel auf das Kalenderjahr. Für einen Vergleich mit den zentralen makroökonomischen Größen ist daher ausschließlich die jahresbezogene Tariferhöhung mit Bezug auf das Vorjahr methodisch zulässig und verwendbar. Nur die Erhöhung der Tarifentgelte des Berichtsjahres im Vergleich zum Vorjahr kann beispielsweise sinnvoll zur Veränderungsrate des Bruttoinlandsprodukts (BIP), 
der Lebenshaltungskosten, der Arbeitsproduktivität, der Gewinne u. ä. in Beziehung gesetzt werden, da diese ebenfalls auf Jahresbasis ermittelt werden (vgl. genauer Bispinck 2011).

Die jahresbezogene Steigerung der Tarifverdienste bringt - anders als die Abschlussrate - die Steigerung des durchschnittlichen tariflichen Monatsentgelts des laufenden Jahres gegenüber dem Vorjahr zum Ausdruck. Einbezogen werden bei dieser Größe auch die Pauschalzahlungen z. B. für verzögert in Kraft getretene Neuabschlüsse und zusätzliche Einmalzahlungen.

Auf der Basis der vorliegenden diesjährigen Abschlüsse (Stichtag 30.6.2012) beträgt die kalenderjährliche Erhöhung der Tarifverdienste für 2012 durchschnittlich 3,0 \%. Für jene Wirtschaftszweige und Tarifbereiche, für die bereits im vergangenen Jahr oder früher Tarifanhebungen für das gesamte Jahr 2012 vereinbart wurden, errechnet sich eine jahresbezogene Steigerung von $2,5 \%$.

Insgesamt ergibt sich für 15,3 Mio. Beschäftigte eine durchschnittliche Tarifsteigerung für das Jahr 2012 von 2,7\%. Dieses Gesamtergebnis für 2012 wird also durch die Abschlüsse aus den Vorjahren deutlich nach unten gedrückt, die für immerhin 8,2 Mio. Beschäftigte gelten, während die Neuabschlüsse in diesem Jahr bislang 7,1 Mio. Beschäftigte betreffen (Tabelle 1).

Die kalenderjährliche Tarifsteigerung von voraussichtlich 2,7 \% für dieses Jahr liegt deutlich über der Höhe des Vorjahres (2011: 2,0 \%). Angesichts der geschätzten Preissteigerungsrate von jahresdurchschnittlich knapp $2 \%$ wird mit dieser Tariferhöhung gesamtwirtschaftlich in diesem Jahr eine merkliche tarifliche Reallohnsteigerung erreicht. Im Jahr 2011 war noch eine tarifliche Reallohnsenkung zu verzeichnen gewesen (Bispinck/WSI-Tarifarchiv 2012).

Zwischen den einzelnen Wirtschaftsbereichen ergeben sich allerdings deutliche Unterschiede. Die Spannweite variiert zwischen 1,9 \% im Verbrauchsgütergewerbe und 3,3\% im Investitionsgütergewerbe. Dazwischen liegen beispielsweise die Bereiche Energie- und Wasserversorgung, Bergbau sowie Kreditinstitute, Versicherungsgewerbe mit 2,0 \%, der Bereich Gebietskörperschaften, Sozialversicherung mit 2,2 \%, das Baugewerbe und der Bereich Verkehr und Nachrichtenübermittlung mit 2,4\%. Genau im Durchschnitt liegt der Handel mit 2,7 \%. Und über dem Durchschnitt liegen der Bereich Gartenbau, Land- und Forstwirtschaft mit 2,9 \%, das Grundstoff- und Produktionsgütergewerbe sowie das Nahrungs- und Genussmittelgewerbe mit 3,0 \%.

Der Unterschied zwischen der tariflichen Abschlussrate für 2012 von 3,9\% und der jahresbezogenen Tarifsteigerung von 2,7 \% geht im Wesentlichen auf zwei Faktoren zurück: Zum einen treten die Tarifanhebungen häufig erst im Laufe dieses Jahres in Kraft und wirken damit nicht auf das gesamte Kalenderjahr (dies gilt gleichermaßen für die Pauschalzahlungen für Verzögerungsmonate), zum anderen gibt es Basiseffekte aus dem Vorjahr. So haben z. B. Pauschalzahlungen eine senkende Wirkung: Während sie die Summe der Tarifvergütungen im Vorjahr erhöhen, entfallen sie im Folgejahr.
Das Tarifniveau Ost/West hat sich im vergangenen halben Jahr praktisch nicht verändert, es beträgt für die tariflichen Grundvergütungen der mittleren Gruppe auf der Basis von Tarifbereichen mit 1,6 Mio. erfassten Beschäftigten zur Jahresmitte 2012 96,3\% (Ende 2011: 96,5\%).

\section{Ausgewählte Tarifrunden}

Die Tarifrunden im öffentlichen Dienst, in der Metallindustrie und in der chemischen Industrie haben die Tarifrunde 2012 maßgeblich geprägt. Sie werden im Folgenden in der zeitlichen Abfolge der Verhandlungen in Verlauf und Ergebnis dargestellt und analysiert.

\section{1 Öffentlicher Dienst - Bund und Gemeinden}

\subsubsection{Ausgangssituation und Forderungen}

Die Gewerkschaften des öffentlichen Dienstes bemühen sich seit Jahren darum, den vor allem seit 2005 entstandenen Rückstand gegenüber der durchschnittlichen Tarifentwicklung in den anderen Wirtschaftsbereichen wieder wettzumachen. Dies gelingt in unterschiedlichem Maße und gestaltet sich wegen der problematischen Lage der öffentlichen Finanzen, namentlich im Bereich der Gemeinden, als sehr schwierig.

Verhandelt wird seit Jahren getrennt für die Bereiche Bund und Gemeinden bzw. für die Länder. In diesem Jahr stand der Tarifbereich Bund und Gemeinden im Tarifkalender. Der Tarifvertrag lief Ende Februar 2012 aus. Der letzte Abschluss für diesen Bereich war mit einer Laufzeit von 26 Monaten im Februar 2010 vereinbart worden und umfasste eine Tarifanhebung in drei Stufen: 1,2 \% ab Januar 2010, weitere 0,6 \% ab Januar 2011 und 0,5\% ab August 2011. Für die Länder hat es den letzten Abschluss im März 2011 gegeben, der bei einer Laufzeit von 24 Monaten u. a. eine Pauschalzahlung sowie eine Tarifanhebung in zwei Schritten von 1,5\% und 1,9\% und anschließend $17 €$ vorsah.

Die Tarifrunde war in diesem Jahr relativ kurz: Die Tarifparteien benötigten lediglich vier Wochen und drei Verhandlungsrunden, um zu einem Ergebnis zu kommen. Die Schlichtung wurde, anders als in vielen Runden zuvor, nicht angerufen. Dies hing zweifelsohne mit dem erheblichen Druck zusammen, den die Gewerkschaften durch die umfangreichen Warnstreiks entfalteten.

Neben ver.di zählen die Gewerkschaft Erziehung und Wissenschaft (GEW), die Eisenbahn- und Verkehrsgewerkschaft (EVG) und die Gewerkschaft der Polizei (GdP) seitens des Deutschen Gewerkschaftsbundes (DGB) gemeinsam mit der dbb Beamtenbund und Tarifunion zu den verhandelnden Gewerkschaften im öffentlichen Dienst. 


\section{Tarifsteigerung 2012 $^{1}$}

\begin{tabular}{|c|c|c|c|c|c|c|}
\hline \multirow[t]{2}{*}{ Wirtschaftsbereich } & \multicolumn{2}{|c|}{ West } & \multicolumn{2}{|c|}{ Ost } & \multicolumn{2}{|c|}{ Gesamt } \\
\hline & AN in 1000 & $\%$ & AN in 1000 & $\%$ & AN in 1000 & $\%$ \\
\hline Gartenbau, Land- und Forstwirtschaft & 57,1 & 2,8 & 17,3 & 3,3 & 74,4 & 2,9 \\
\hline Energie- und Wasserversorgung, Bergbau & 107,2 & 1,7 & 22,9 & 3,4 & 130,1 & 2,0 \\
\hline Grundstoff- und Produktionsgütergewerbe & 749,0 & 3,0 & 76,8 & 3,1 & 825,8 & 3,0 \\
\hline Investitionsgütergewerbe & $3.680,3$ & 3,3 & 385,8 & 3,4 & $4.066,1$ & 3,3 \\
\hline Verbrauchsgütergewerbe & 388,9 & 1,8 & 96,4 & 2,4 & 485,3 & 1,9 \\
\hline Nahrungs- und Genussmittelgewerbe & 234,9 & 2,9 & 36,4 & 3,4 & 271,3 & 3,0 \\
\hline Baugewerbe & 634,2 & 2,4 & 173,3 & 2,7 & 807,5 & 2,4 \\
\hline Handel & $2.855,5$ & 2,7 & 421,3 & 2,7 & $3.276,8$ & 2,7 \\
\hline Verkehr und Nachrichtenübermittlung & 735,6 & 2,3 & 109,6 & 2,7 & 845,2 & 2,4 \\
\hline Kreditinstitute, Versicherungsgewerbe & 388,3 & 2,0 & 30,7 & 2,0 & 419,0 & 2,0 \\
\hline Private Dienstleistungen, Org. o. Erwerbszweck & $1.555,3$ & 2,4 & 426,8 & 3,1 & $1.982,1$ & 2,5 \\
\hline Gebietskörperschaften, Sozialversicherung & $1.654,3$ & 2,2 & 460,9 & 2,1 & $2.115,2$ & 2,2 \\
\hline Gesamte Wirtschaft & $13.040,6$ & 2,7 & $2.258,2$ & 2,8 & $15.298,8$ & 2,7 \\
\hline
\end{tabular}

1 Jahresbezogene Erhöhung 2012 gegenüber 2011

Quelle:WSI-Tarifarchiv 2012; Stand: 30.06.2012.

Nach einer intensiven Vordiskussion beschloss die Bundestarifkommission von ver.di am 9.2.2012 folgenden Forderungskatalog:

- Erhöhung der Tarifentgelte im Bereich des Tarifvertrages für den Öffentlichen Dienst (TVöD) und des TV-N (Nahverkehr) um 6,5\%, mindestens aber $200 €$ im Monat; im Bereich des TV-V (Versorgungsbetriebe) Anhebung um $7,9 \%$;

- Erhöhung der Ausbildungsvergütungen um $100 €$;

- Laufzeit von zwölf Monaten;

- unbefristete Übernahme der Auszubildenden nach erfolgreich abgeschlossener Ausbildung im erlernten Beruf;

- Flughäfen: Zulage in Höhe von $90 €$ monatlich zum Ausgleich von Belastungen durch Safety und Security;

- zusätzliche Forderungen für die Bereiche Theater und Bühnen sowie Sparkassen.

Die zentrale Begründung der Forderungen von ver.di stand unter dem Motto „Du bist es wert“ und griff damit den Grundtenor der diesjährigen tarifpolitischen Debatte auf, dass nach Jahren der Zurückhaltung die Beschäftigten nunmehr Anspruch auf faire Teilhabe an der wirtschaftlichen Entwicklung hätten. Ver.di und die anderen Gewerkschaften postulierten einen Nachholbedarf gegenüber der Privatwirtschaft, ein Abschlag für den öffentlichen Dienst sei nicht gerechtfertigt. Deutliche Entgeltsteigerungen seien auch ein Gebot der wirtschaftlichen Vernunft. Ver.di-Vorsitzender Frank Bsirske konstatierte für die Beschäftigten des öffentlichen Dienstes einen Re- allohnverlust im Jahr 2011 von 0,6 \% und damit einen weiter vergrößerten Abstand zu den Einkommen der Privatwirtschaft (ver.di-Presseinformation vom 9.2.2012). Zur Finanzierbarkeit der Forderungen argumentierte ver. di, dass die schwierige Haushaltssituation nicht naturgegeben, sondern maßgeblich durch politische Entscheidungen herbeigeführt worden sei. Mit einem Konzept zur Steuergerechtigkeit habe die Gewerkschaft Vorschläge zur notwendigen Verbesserung der öffentlichen Einnahmen gemacht (ver.di 2011).

Die Gewerkschaften legten die Verhandlungen so an, dass bereits für die erste Verhandlungsrunde am 1.3.2012 von den öffentlichen Arbeitgebern ein verhandlungsfähiges Angebot verlangt wurde. Zur Unterstützung dieser Forderungen wurde in den Tagen unmittelbar zuvor eine Vielzahl von gewerkschaftlichen Aktionen durchgeführt. Die Arbeitgeber wiesen die gewerkschaftliche Forderung als „,öllig unrealistisch“ zurück und machten die Vorlage eines Angebots von der Reduzierung der Forderungen abhängig. Die kommunalen Arbeitgeber wiesen vor allem auf die Rekordverschuldung der Kommunen hin. Ein Nachholbedarf des öffentlichen Dienstes wurde bestritten. Die Mindestforderung von $200 €$ wurde strikt abgelehnt. Sie betreffe $62 \%$ der Entgeltgruppen und keinesfalls nur die unteren Gruppen. Sie untergrabe auch die Wettbewerbsfähigkeit der kommunalen Unternehmen. Die Gewerkschaften riefen daraufhin zu Warnstreiks in der Woche ab dem 5.3. auf, an denen sich rund 130.000 Beschäftigte beteiligten. Ver.di wertete dies als sehr gute Beteiligung, die deutlich über den Erwartungen gelegen habe. 
In der zweiten Verhandlungsrunde am 12./13.3.2012 legten Bund und kommunale Arbeitgeber ein erstes Angebot vor, das folgende Elemente umfasste:

- Anhebung der Tarifentgelte um 2,1\% ab 1.5.2012,

- Stufenanhebung von weiteren $1,2 \%$ ab 1.3.2013,

- Pauschalzahlung von $200 €$ (Auszubildende $40 €$ ) für März und April,

- Laufzeit von 24 Monaten,

- Übernahme der Auszubildenden für zwölf Monate bei entsprechendem Bedarf.

In der öffentlichen Darstellung stellten die Arbeitgeber die Erhöhung um 3,3 \% in der Summe heraus und reklamierten, dass die Beschäftigten im öffentlichen Dienst damit an der allgemeinen Lohnentwicklung teilhaben würden. Aus Sicht der Gewerkschaften war dieses Angebot „in keinem Punkt akzeptabel“. Ver.di errechnete unter Berücksichtigung der beiden Leermonate und der Pauschalzahlung eine durchschnittliche Erhöhung von 1,77 \% je Jahr und damit Reallohnverluste. Die Einmalzahlung könne nicht als soziale Komponente angesehen werden und die Übernahmeregelung schreibe lediglich die unzureichende Regelung aus dem Jahr 2010 fest.

\subsubsection{Ergebnis}

Um den Druck auf die Arbeitgeberseite zu erhöhen, riefen die Gewerkschaften ab dem 19.3. zu einer zweiten, ausgeweiteten Warnstreikwelle auf. Mit insgesamt 215.000 Teilnehmenden beteiligten sich noch einmal deutlich mehr Beschäftigte als an der ersten Warnstreikwelle. Die dritte Verhandlungsrunde war für den 28./29.3. terminiert und dauerte mit mehreren Unterbrechungen bis zum Morgen des 31.3. Sie führte letztlich zu folgendem Ergebnis:

- Erhöhung der tariflichen Entgelttabellen rückwirkend ab 1.3.2012 um 3,5\%;

- Stufenerhöhung um 1,4 \% ab 1.1.2013 und weitere 1,4 \% ab 1.8.2013;

- Laufzeit von insgesamt 24 Monaten bis Ende Februar 2014;

- Erhöhung der Ausbildungsvergütungen ab März 2012 um $50 €$ und ab August 2013 um weitere $40 €$;

- Übernahme der Ausgebildeten nach bestandener Prüfung für zunächst zwölf Monate, bei Bewährung und Bedarf danach unbefristet;

- Flughäfen: Sonderzahlung von $600 €$ für Beschäftigte an Flughäfen mit mindestens 5 Mio. Passagieren und $200 €$ für alle übrigen

- Urlaub: ab 2013 Urlaubsanspruch von 29 Arbeitstagen für alle Beschäftigte, nach dem 55. Lebensjahr von 30 Tagen. Besitzstandsregelung für alle mit derzeit 30 Tagen Urlaubsanspruch. ${ }^{2}$

Materiell bedeutet dieser Abschluss, dass die Tabellenvergütungen nach Umsetzung der drei vereinbarten Anhe- bungen um 6,4 \% über dem Ausgangsniveau liegen. Jahresbezogen ergibt sich für das Jahr 2012 eine Erhöhung der Tarifvergütung gegenüber dem Vorjahr um 2,4 \% und für das Jahr 2013 gegenüber 2012 um 2,6 \%. Die Ausbildungsvergütungen werden überproportional um insgesamt $10-12 \%$ angehoben. Positiv wurde die Einmalzahlung für die Beschäftigten an Flughäfen bewertet. Die Regelung zur Ausbildungsübernahme blieb allerdings deutlich hinter den Erwartungen zurück.

Dieses Ergebnis wurde in der Bundestarifkommission von ver.di lange und intensiv diskutiert. Insbesondere das Fehlen einer sozialen Komponente wurde heftig kritisiert. Auch die Urlaubsregelung fand nur begrenzte Zustimmung. Letztlich stimmte die Bundestarifkommission dem Ergebnis dann doch mehrheitlich zu, vor allem deswegen, weil angesichts der vereinbarten Entgeltzuwächse für wichtige Arbeitskampfbereiche keine hinreichende Streikbereitschaft mehr erwartet wurde. In der bis zum 24.4.2012 durchgeführten Befragung sprachen sich $74 \%$ der betroffenen Mitglieder für die Annahme der Tarifeinigung aus. Das Diskussionsspektrum markieren zwei unterschiedliche Einschätzungen prominenter ver.di-Funktionäre: Der frühere stellvertretende ver.di-Bezirksleiter in Bayern, Michael Wendl, konstatierte einen tarifpolitischen Erfolg, weil der Abschluss den Anforderungen an eine produktivitätsorientierte Lohnpolitik gerecht werde und ohne Schlichtung erreicht worden sei (Wendl 2012). Eine kritischere Bewertung nahm dagegen der Stuttgarter ver.di-Geschäftsführer Bernd Riexinger vor. Er kritisierte den mehrfachen Wechsel in der Verhandlungsführung und insbesondere den Verzicht auf die Umsetzung der von ver.di geplanten Erzwingungsstreikstrategie (Riexinger 2012).

Ver.di sprach von einem insgesamt ,beachtlichen Ergebnis, das im Wesentlichen der Streikbereitschaft der Beschäftigten zu verdanken gewesen sei“. Es sei mit dem Abschluss gelungen, die Reallöhne für 2012 und 2013 nachhaltig zu sichern", betonte der ver.di-Vorsitzende Frank Bsirske. Aus Sicht der Arbeitgeber handelte es sich um einen „schwierigen, aber gerade noch vertretbaren Abschluss". Als Pluspunkte nannten die Arbeitgeber die Laufzeit von 24 Monaten mit stufenweisen Erhöhungen, das Vermeiden eines Mindestbetrages und die Neuregelung des Urlaubsanspruchs. Außerdem habe ein Flächenstreik vermieden werden können.

In der Presse wurde der Abschluss unterschiedlich kommentiert. Die Frankfurter Allgemeine (FAZ) befand, der „Tarifabschluss kommt den Staat teuer zu stehen“. Es sei „unerklärlich“, warum die öffentlichen Arbeitgeber „so schnell und so stark eingeknickt" seien (2.4.2012). Die Fi-

(2) Eine neue Urlaubsregelung war notwendig geworden, weil das Bundesarbeitsgericht am 20.3.2012 entschieden hatte, dass die bestehende Urlaubsstaffelung (26 Arbeitstage unter 30, 29 unter 40 Lebensjahren und 30 ab dem 40. Lebensjahr) gegen das Allgemeine Gleichbehandlungsgesetz (AGG) verstößt. 
nancial Times Deutschland sah voraus, dass das "satte Plus“ auch die Verhandlungen in der Industrie beeinflussen werde und zitierte Bundesbank-Präsidenten Jens Weidmann mit der Warnung, den Abschluss als Vorbild für andere Branchen zu nehmen (2.4.2012). Die Frankfurter Rundschau machte hingegen darauf aufmerksam, dass die Volkswirte der Banken den Lohnabschluss lobten, weil er eine gute Nachricht für den privaten Konsum sei und dazu beitrage, die Ungleichgewichte in Europa zu verringern (3.4.2012).

\subsection{Metall- und Elektroindustrie}

\subsubsection{Ausgangslage und Forderungen}

Die Metall- und Elektroindustrie bestimmte dieses Jahr maßgeblich das Tarifgeschehen in der privaten Wirtschaft. Der Tarifabschluss vom Februar 2010 lief Ende März dieses Jahres aus. Er war noch unter dem Eindruck der Finanzund Wirtschaftskrise abgeschlossen worden und beinhaltete u. a. detaillierte Regelungen zur tariflichen Kurzarbeit und Beschäftigungssicherung (Bispinck/WSI-Tarifarchiv 2011). Im materiellen Teil umfasste er eine Einmalzahlung von insgesamt $320 €$ für elf Monate und eine anschließende Tariferhöhung von 2,7 \% ab April 2011 für weitere zwölf Monate. Auf die überraschend starke wirtschaftliche Erholung im Laufe des Jahres 2010 und 2011 konnte die IG Metall aufgrund der Laufzeit tarifpolitisch nicht unmittelbar reagieren. Allerdings gab es auf betrieblicher Ebene zahlreiche Aktivitäten, um die tariflich vereinbarte Möglichkeit des Vorziehens der Tariferhöhung um zwei Monate auch praktisch zu nutzen. Nach einer Erhebung der IG Metall in Baden-Württemberg geschah dies in etwa $30 \%$ der Betriebe mit etwas mehr als $50 \%$ der Beschäftigten. Eine Reihe von Firmen zahlte zusätzliche Einmalzahlungen und Boni, sodass sich 2011 in der Metallindustrie, aber auch in anderen Branchen, eine spürbar positive Lohndrift entwickelte.

Die IG Metall wollte die Tarifrunde 2012 auch für zwei qualitative Themen nutzen: Die Regulierung der Leiharbeit und die Übernahme der Ausgebildeten. Zu beiden Themen hatte die Gewerkschaft bereits vor einiger Zeit Kampagnen gestartet, die auf betrieblicher, tarifpolitischer und gesellschaftspolitischer Ebene die Forderungen der IG Metall bekannt machen und verankern sollten. Unter der Überschrift „Leiharbeit fair gestalten“ zielt die Initiative „Gleiche Arbeit - Gleiches Geld“ der IG Metall seit ihrem Start im April 2008 im Kern auf die Durchsetzung des Equal PayGrundsatzes für die Leiharbeit. ${ }^{3}$ Die „Operation Übernahme" wiederum zielte auf die unbefristete Übernahme der Ausgebildeten durch den ausbildenden Betrieb. Im Januar 2009 hatte die IG Metall-Jugend diese Kampagne auf den Weg gebracht. In den bezirklichen Tarifkommissionen der IG Metall wurden beide Themen bereits im Frühjahr 2011 angesprochen. In beiden Bereichen sollte mit den Arbeitgebern schon im Vorfeld der Tarifrunde gesprochen werden, um auszuloten, ob und welche Gestaltungsmöglichkeiten bestünden.

Die Diskussion über die möglichen materiellen Forderungen in der Entgeltrunde hatte die IG Metall zeitlich nach hinten geschoben, weil sich im Laufe des Jahres 2011 die wirtschaftlichen Perspektiven eintrübten (zur Analyse der Branchenentwicklung IG Metall 2012). Um möglichst große Sicherheit über die absehbare Branchenentwicklung zu gewinnen, sprach der Vorstand der IG Metall erst am 7.2.2012 seine Forderungsempfehlung aus. Er empfahl bis zu 6,5 \% höhere Löhne, Gehälter und Ausbildungsvergütungen für eine Laufzeit von zwölf Monaten. Diese Forderung wurde dann am 24.2.2012 auch offiziell bestätigt.

Bei der Höhe der Forderung legte die IG Metall nach eigenen Angaben die gesamtwirtschaftliche Produktivitätsund Inflationsentwicklung zugrunde (IG Metall 2012, S. 10). „Wirtschaftliche Zuwächse und die Entgelterhöhungen müssen sich mittelfristig parallel entwickeln“, sagte Helga Schwitzer, geschäftsführendes Vorstandsmitglied der IG Metall. Deshalb bilanziere die IG Metall vor dem Hintergrund des letzten Abschlusses auch die reale Entwicklung im Jahr 2011. Das vergangene Jahr sei ein überaus erfolgreiches Jahr für die Metall- und Elektroindustrie gewesen.

Die Gewerkschaft bekräftigte die beiden qualitativen Forderungen: Zum einen sollte die unbefristete Übernahme der Ausgebildeten in den Betrieben zur Regel gemacht und die Ausbildungsfähigkeit benachteiligter Jugendlicher gefördert werden. Zum anderen forderte die IG Metall mehr Mitbestimmungsrechte von Betriebsräten bei Dauer, Einsatz und Umfang von Leiharbeit. Der IG Metall-Vorsitzende Berthold Huber betonte, es handele sich um drei gleichwertige Forderungen. „Im Zweifel werden wir einen Konflikt um alle drei Themen führen“ (IG Metall Pressemeldung vom 24.2.2012).

Die Metallarbeitgeber reagierten bereits auf die Forderungsempfehlung mit strikter Ablehnung. Die 6,5\% seien nicht nachvollziehbar. Die IG Metall könne „maximal $3 \%$ mit wirtschaftlichen Kennziffern belegen“. Für die restlichen 3,5 \% gebe es keine belastbare Grundlage. Die Tarifentgelte seien seit der Krise um $9 \%$ gestiegen, die Verbraucherpreise dagegen lediglich um 6,5\%, für die Beschäftigten habe sich also ein reales Plus ergeben. Gesamtmetall-Präsident Martin Kannegießer bezeichnete die geforderte Ausweitung der Mitbestimmung bei der Leiharbeit als „,nicht einsichtig, schädlich und deshalb inakzeptabel“. Branchenzuschläge für die Leiharbeitsbeschäftigten seien im Grundsatz zweckmäßig und nachvollziehbar. Damit müsste dann das Thema aber

3 Ein gesetzlicher Mindestlohn sollte zudem Leiharbeit zu Armutslöhnen verhindern, das Synchronisationsverbot sollte wieder eingeführt werden, um das "Heuern und Feuern“ zu unterbinden, und eine Höchstverleihzeit sollte verhindern, dass Leiharbeit als Instrument zum Abbau von StammArbeitsplätzen genutzt wird (http://www.gleichearbeitgleichesgeld.de/initiative/gruendungserklaerung/). 
„endgültig befriedet" werden. Die eingeschränkte externe Flexibilität müsste durch eine Ausweitung der internen Flexibilität ausgeglichen werden, konkret: durch eine Ausweitung der sachgrundlosen Befristung oder eine Erhöhung der Quote von Beschäftigten mit verlängerter tariflicher Arbeitszeit (Gesamtmetall Presseerklärung 03/2012 vom 7.2.2012).

\subsubsection{Verhandlungen}

Die Tarifverhandlungen begannen am 6.3.2012 für die Beschäftigten der Tarifgebiete Baden-Württemberg, Niedersachsen, der Mittelgruppe (Hessen, Rheinland-Pfalz, Saarland) und Thüringen sowie am 9. März in Bayern. Die übrigen Bezirke folgten zwischen dem 14. und 20.3. Die erste Runde diente dem üblichen Austausch über die wirtschaftliche Situation der Branche und der Gesamtwirtschaft sowie der Präsentation der Tarifforderungen und endete erwartungsgemäß ohne Ergebnis. Der Auftakt der zweiten Runde fand am 21.3.2012 in Niedersachsen statt, schrittweise gefolgt von den anderen Bezirken bis zum 5.4.2012. Die Arbeitgeber legten auch in dieser Runde kein Angebot vor. Die IG Metall präzisierte in Baden-Württemberg ihre Vorstellungen zur Übernahme. Der Lösungsvorschlag enthielt auch Ausnahmen von der unbefristeten Übernahme. In der dritten Verhandlungsrunde, die am 18.4. in Nordrhein-Westfalen begann, legten die Arbeitgeber dann ein erstes Angebot vor, das folgende Elemente beinhaltete:

- Erhöhung der Tarifentgelte um 3,0 \% ab 1.4.2012 mit einer Laufzeit von 14 Monaten;

- Empfehlung an die Mitgliedsunternehmen zur unbefristeten Übernahme der Ausgebildeten;

- keine Regelungen zur erweiterten Mitbestimmung bei der Leiharbeit;

- Erhöhung der Quote für Beschäftigte mit dauerhafter Arbeitszeitverlängerung auf bis zu 40 Stunden um zwölf Prozentpunkte;

- Ausweitung der Dauer der sachgrundlosen Befristung von 24 auf 36 Monate.

Die IG Metall lehnte das Angebot ab, u. a., da die Erhöhung auf ein Jahr umgerechnet noch nicht einmal eine Reallohnsicherung bedeutet hätte und zu den Themen Übernahme und Zeitarbeit kein Angebot vorgelegt wurde. Auch in den nachfolgenden regionalen Verhandlungen gingen die Arbeitgeber nicht über dieses Angebot hinaus. Am 8.5. begann in Baden-Württemberg die vierte Verhandlungsrunde, die ohne ein neues Angebot endete. Auf das Verhalten der Arbeitgeber reagierte die IG Metall nach Ablauf der Friedenspflicht mit einer massiven Ausweitung der Warnstreiks. Bis einschließlich 9.5. beteiligten sich bundesweit insgesamt fast 500.000 Beschäftigte an Warnstreiks und Aktionen, um so den Druck auf die Arbeitgeber zu erhöhen. In der fünften Verhandlungsrunde konnten am 16.5. erste Fortschritte bei den Themen Übernahme und Leiharbeit erreicht werden. Die Verhandlung wurde zur Beratung unterbro- chen und am 18. Mai wieder aufgenommen. In den sich anschließenden Verhandlungen verständigten sich IG Metall und Arbeitgeber am 19.5. auf ein Ergebnis, das im Zeitraum bis zum 29.5. im Wesentlichen in den anderen Tarifgebieten übernommen wurde.

\subsubsection{Ergebnis}

Entgelt: Materiell wurde eine Anhebung der Tarifentgelte nach einem Nullmonat (April) um 4,3\% bei einer Laufzeit von insgesamt 13 Monaten vereinbart. Damit vereinbarte die IG Metall erstmals seit dem Jahr 2006 wieder einen Entgeltabschluss mit einer derart kurzen Laufzeit. Die Abschlussrate lag - auch gemessen an der Laufzeit - an der Spitze der bisherigen Abschlüsse der Tarifrunde. Die jahresbezogene Tariferhöhung 2012 gegenüber 2011 beträgt $3,3 \%$. Dies liegt deutlich über der voraussichtlichen Preissteigerung von rund $2 \%$ und bringt den Beschäftigten der Metallindustrie ein erkennbares reales Plus bei den Tarifentgelten. Damit konnten auch die Krisenfolgen bei der Tarifentwicklung zum Teil kompensiert werden. Beide Tarifvertragsparteien werteten dieses Ergebnis positiv.

Übernahme der Ausgebildeten: Folgende Regelungen wurden hierzu vereinbart:

- Die Auszubildenden „sollen“ in der Regel nach bestandener Abschlussprüfung unbefristet in ein Arbeitsverhältnis übernommen werden;

- nach Möglichkeit soll die Anzahl an Ausbildungsplätzen gesteigert, mindestens aber beibehalten werden;

- Möglichkeit einer freiwilligen Betriebsvereinbarung zur Festschreibung des Ausbildungsbedarfs und Festlegung der Anzahl der unbefristet zu übernehmenden Ausgebildeten. Über Bedarf Ausgebildete besitzen keinen Anspruch auf Übernahme;

- wird keine Betriebsvereinbarung vereinbart, beraten Betriebsrat und Arbeitgeber jeweils spätestens sechs Monate vor Ende der Ausbildung über die Anzahl der unbefristet zu übernehmenden Ausgebildeten; über Bedarf Ausgebildete besitzen einen Anspruch auf Übernahme für mindestens zwölf Monate;

- eine Abweichung von der Übernahmeverpflichtung ist möglich, u. a. bei Vorliegen akuter Beschäftigungsprobleme.

Leih-/Zeitarbeit: In einem neuen Tarifvertrag Leih-/Zeitarbeit wurden folgende Regelungen getroffen:

- Der Einsatz von Leiharbeitsbeschäftigten darf im Entleihbetrieb nicht zur Beeinträchtigung der Entgelt-/Arbeitsbedingungen führen und Arbeitsplätze gefährden;

- ein Einsatz ist u. a. zulässig bei zeitlicher Befristung, Vorlage eines Sachgrundes oder Abarbeitung von Auftragsspitzen;

- der Einsatz von Leiharbeitnehmerinnen und Leiharbeitnehmern bedarf grundsätzlich der Zustimmung des Betriebsrats im Entleihbetrieb; 
- ist der Einsatz von Leiharbeitnehmern für länger als drei Monate vorgesehen, muss auf Verlangen des Betriebsrats eine innerbetriebliche Stellenausschreibung erfolgen;

- nach 18/24 Monaten Einsatzdauer: Der Entleiher prüft Möglichkeit des Angebots/grundsätzlich Angebot eines unbefristeten Arbeitsvertrags;

- es erfolgt eine Addition der Einsatzzeiten im selben Betrieb bei Unterbrechungszeiten unter drei Monaten;

- es besteht die Möglichkeit zum Abschluss einer freiwilligen Betriebsvereinbarung, u. a. mit Regelungen zu Einsatzzweck, -bereichen und Volumen von Leiharbeit, Höhe der Vergütung, Einsatzhöchstdauer, Übernahme in ein unbefristetes Arbeitsverhältnis;

- durch eine Betriebsvereinbarung können folgende Möglichkeiten geschaffen werden: Erhöhung der Quote (max. $12 \%$-Punkte) der Arbeitnehmer und Arbeitnehmerinnen mit Arbeitszeit-Verlängerung auf bis zu 40 Std./Woche; im gleichen Volumen Angebot von Arbeitszeit-Reduzierung auf bis zu 30 Std./Woche (verkürzte Vollzeit mit Rückkehrrecht); bei Übernahme von Leiharbeitnehmern Auszahlung von Guthaben aus den Arbeitszeit-Konten;

- der Betriebsrat hat ein Recht auf Information über Umfang und Einsatzbereiche von Leiharbeit sowie auf Einsichtnahme in die Verträge zwischen Arbeitgeber und Verleiher;

- der Entleiher ist dem Verleiher verpflichtet, ihn über betriebliche Regelungen zugunsten der Leiharbeitnehmerinnen und Leiharbeitnehmer zu informieren.

Zum Abschluss gehört schließlich noch eine SozialpartnerVereinbarung „Vom Einstieg zum Aufstieg“, die einen Beitrag zur Stärkung des Fachkräftepotenzials leisten soll. Jugendliche mit Lerndefiziten sollen gefördert, Ausbildungskapazitäten erhalten, gesteigert und die berufliche Entwicklung durch Weiterbildung vorangetrieben werden. Im Rahmen dieser Vereinbarung wurde auch erstmals ein Tarifvertrag „Förderjahr“ abgeschlossen, der konkrete Anforderungen und Maßnahmen für Abschluss und Durchführung eines Fördervertrages mit förderbedürftigen Jugendlichen beschreibt.

Die Bewertung der Regelungen zur Leiharbeit fiel zwischen den Tarifparteien sehr unterschiedlich aus: Die IG Metall stellte heraus, dass die Arbeitgeber ihre Strategie der Totalverweigerung bei Leiharbeit aufgeben mussten. Es sei erstmals gelungen, die Einsatzbedingungen von Leiharbeitern in einem Tarifvertrag zu beschreiben und den Betriebsräten mehr Mitbestimmungsrechte einzuräumen. Das stärke die Position der Betriebsräte gewaltig, so Bezirksleiter Jörg Hofmann (siehe dazu König/Detje 2012). Die Metallarbeitgeber betonten demgegenüber, dass die Betriebe in den ersten zwei Jahren nach wie vor selbst entscheiden könnten, ob und wie sie Leiharbeitsbeschäftigte einsetzen wollten. Aus ihrer Sicht sind vor allem die Bestimmungen zur Arbeitszeitflexibilisierung als Ausgleich für eine eventuelle Beschränkung der Leiharbeit von zentraler Bedeutung.

Branchenzuschläge: Die Frage der Lohnangleichung zwischen Leiharbeitsbeschäftigten und Stammpersonal wurde ab dem 22.2.2012 in insgesamt vier Verhandlungsrunden behandelt. Tarifpartei der IG Metall war die Verhandlungsgemeinschaft Zeitarbeit (VGZ), bestehend aus den beiden Arbeitgeberverbänden der Zeitarbeit, dem Bundesarbeitgeberverband der Personaldienstleister e.V. (BAP, früher BZA) und dem Interessenverband Deutscher Zeitarbeitsunternehmen e.V. (iGZ). In dem am 22.5. abgeschlossenen Tarifvertrag über Branchenzuschläge für Arbeitnehmerüberlassung wurden folgende Bestimmungen vereinbart:

- Nach der 6. Woche bzw. dem 3./5./7./9. Monat ununterbrochener Einsatzdauer in einem Kundenbetrieb wird ein Zuschlag von 15/20/30/45/50 \% auf Basis der Vergütungen der DGB-BZA/iGZ-Tarifverträge gezahlt;

- bei Unterbrechungszeiten unter drei Monaten erfolgt eine Addition der Einsatzzeiten im selben Betrieb;

- der Zuschlag wird auf die Differenz der Vergütung von Leiharbeitsbeschäftigten und vergleichbaren Beschäftigten des Entleihbetriebs ohne Berücksichtigung des Äquivalents einer durchschnittlichen Leistungszulage (10 \%) begrenzt; - Leiharbeitnehmerinnen und Leiharbeitnehmer erhalten den Branchenzuschlag auch, wenn sie in nicht tarifgebundenen Metall- und Elektrounternehmen arbeiten;

- Wegfall der einsatzbezogenen Zulage gemäß $\$ 4$ ETV bzw. $\$ 5$ ERTV zwischen DGB und BZA bzw. iGZ;

- Anspruch auf Leistungen entsprechend den betrieblichen Vereinbarungen im Entleihbetrieb;

- Verfahrensregelung zur Anpassung des Branchenzuschlags an Tariferhöhungen;

- die Tarifparteien gehen davon aus, dass im Laufe der vereinbarten Erklärungsfrist weitere DGB-Gewerkschaften entsprechende Tarifverträge über Branchenzuschläge abschließen.

Die IG Metall, so heißt es in dem Abkommen, werde auf die anderen DGB-Gewerkschaften innerhalb der Tarifgemeinschaft Zeitarbeit entsprechend Einfluss nehmen und sie über das Verfahren zur Ermittlung der Branchenzuschläge informieren. ${ }^{\circledR}$

Der Tarifvertrag soll am 1. November 2012 in Kraft treten und hat eine Laufzeit bis Ende 2017. Die IG Metall bezifferte den Zuschlag für Leiharbeitnehmer in der untersten Entgeltgruppe je nach Einsatzdauer zwischen 186,33€ und $621,09 €$ pro Monat (Abbildung 1) und wertete den Vertrag als einen „wichtigen Schritt hin zur fairen Bezahlung" von Leiharbeitsbeschäftigten. Die IG Metall wies darauf hin, dass unabhängig von diesen tarifpolitischen Fortschritten der Gesetzgeber in Sachen Re-Regulierung der Leiharbeit nach wie vor gefordert bleibt (vgl. auch Burmeister 2012).

(4) Tatsächlich haben einige Gewerkschaften Sondierungsgespräche bzw. Verhandlungen mit BAP/iGZ aufgenommen. Es gab aber auch kritische Stimmen aus den Gewerkschaften zu dem Abschluss (vgl. ver.di 2012). 
ABB. 1

\section{Tarifliches Grundentgelt der untersten Entgeltgruppe* in Leih-/ Zeitarbeit (BAP/iGZ) und Metallindustrie Baden-Württemberg}

Anteile in Prozent

Grundentgelt Metall

Grundentgelt BAP/iGZ

- Branchenzuschlag

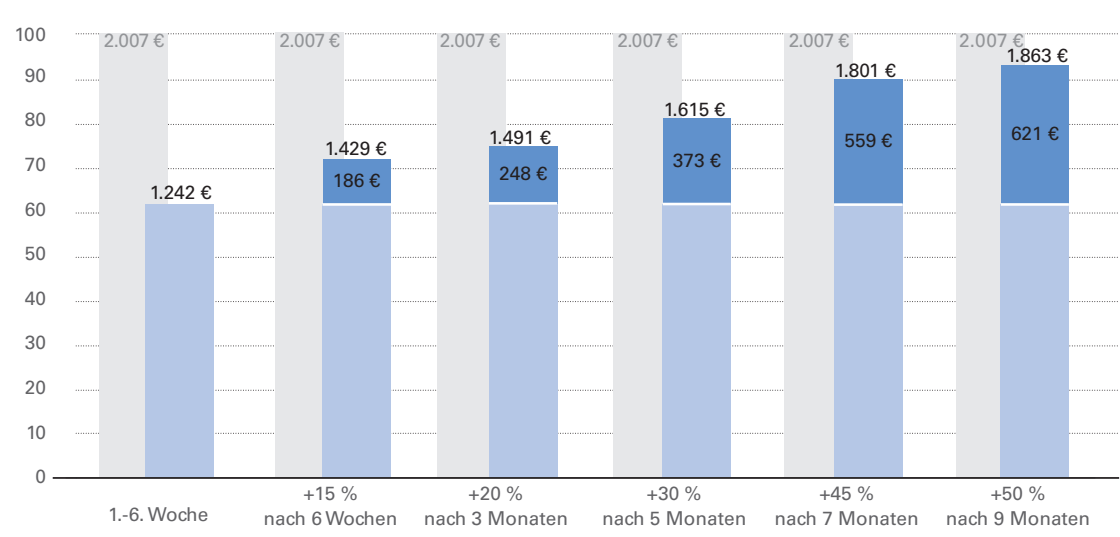

Beschäftigungszeit

*Gerundete Beträge, Rundungsdifferenzen möglich. Regelungsstand: 1.11.2012.

Quelle: Berechnungen WSI-Tarifarchiv.
Vorsitzende Michael Vassiliadis (IG BCE-Medieninformation vom 14.2.2012). Die Herausforderungen des demografischen Wandels ließen sich keinesfalls mit einer simplen Ausdehnung der Arbeitszeit bewältigen. Schwerpunkte der Arbeitszeitpolitik sollten bei der Entlastung in bestimmten Lebensphasen und bei der Gestaltung attraktiver Arbeitsbedingungen für Berufseinsteiger liegen, so der IG BCE-Tarifvorstand Peter Hausmann (ebd.). Die Forderungsempfehlung war Grundlage für Diskussionen in den Betrieben, deren Ergebnisse in den regionalen Tarifkommissionen zusammengeführt wurden. Am 3.4. beschloss die Bundestarifkommission der IG BCE die Forderung endgültig unter dem Motto „Gute Arbeit - Faires Entgelt“. Die Arbeitgeber hielten sich in ihrer Stellungnahme bemerkenswert zurück. Der Bundesarbeitgeberverband Chemie (BAVC) empfahl mit Blick auf die Entgeltforderung „Realismus als Richtschnur“. Zu den Realitäten gehörten auch die konjunkturelle Abschwächung, die Unsicherheit durch die Schuldenkrise und die hohe Volatilität in den Märkten. An der Spitze der TarifAgenda stehe die demografische Frage. Neben mehr Flexibilität für die Beschäftigten in verschiedenen Lebenslagen müsse mehr Flexibilität für die Unternehmen ermöglicht werden, etwa durch die Verlängerung der tariflichen Arbeitszeit. Die altersbezogenen Tarifregelungen (z. B. Altersfreizeiten ab 55 und Verdienstsicherung ab 50) sollten auf den Prüfstand gestellt werden (BAVC-Presseinformation vom 14.2.2012).

Traditionell begannen die Verhandlungen mit einer regionalen Runde im Zeitraum vom 17.4. (Hessen) und 26.4. (Westfalen), die im Wesentlichen dem Austausch der grundsätzlichen Positionen diente. Die erste Verhandlungsrunde auf Bundesebene fand am 7.5.2012 statt. In der Entgeltfrage stellten die Arbeitgeber heraus, dass die Beschäftigten bereits 2011 mit dem „deutschlandweit höchsten Tarifabschluss" von 4,1 \% am Erfolg der Branche beteiligt worden seien, einen Nachholbedarf gebe es nicht. Die Chemieproduktion werde stagnieren, die Branche müsse höhere Rohstoffkosten und steigende Energiepreise verkraften. Ein konkretes Entgeltangebot legten sie weder regional noch auf Bundesebene vor.

Auch in der Arbeitszeitfrage blieben die Auffassungen konträr. IG BCE und BAVC verständigten sich allerdings auf die Einsetzung von Arbeitsgruppen, um bei der Weiterentwicklung des Tarifvertrags zur Lebensarbeitszeit und Demografie Möglichkeiten einer Einigung zu erörtern. Die IG BCE wertete dies als „Fortschritt“, aber in der Sache lägen die Positionen noch weit auseinander. In den folgenden zwei Wochen gab es auf betrieblicher und lokaler Ebene zahlreiche Protestveranstaltungen und Demonstrationen zur Unterstützung der Gewerkschaftsforderungen, an denen sich tausende Beschäftigte beteiligten. In der zweiten bundesweiten Verhandlungsrunde am 23. und 24.5. in BerlinSchönefeld gelang nach langen und schwierigen Verhandlungen eine Einigung:
„Viele Betriebe strotzen vor Kraft, die Wettbewerbsfähigkeit hat ein außerordentlich hohes Niveau", so der IG BCE-
Die Einkommen in der chemischen Industrie sollten danach um 6 \% erhöht werden bei einer Laufzeit von zwölf Monaten. Zur Begründung verwies die Gewerkschaft darauf, dass sich nach dem Rekordjahr 2011 die Lage in der 


\subsubsection{Entgelt und Ausbildungsvergütungen}

- Die Entgelte werden nach einem Nullmonat um 4,5 \% angehoben, die Laufzeit beträgt insgesamt 19 Monate. Die Ausbildungsvergütungen werden einheitlich um $50 €$ erhöht. - Die Tariferhöhung tritt regional unterschiedlich in Kraft: in den Tarifbezirken Nordrhein, Rheinland-Pfalz und Hessen zum 1.7., in Westfalen, Bayern, Baden-Württemberg, Niedersachsen/Bremen, Schleswig-Holstein/Hamburg und Berlin zum 1.8. und im Saarland und in Nordost zum 1.9.

- In Betrieben mit besonderem wirtschaftlichem Erfolg kann die Tariferhöhung im Einvernehmen der Betriebsparteien um einen Monat vorgezogen werden. Als Indizien gelten Gewinnausschüttung oder erfolgsbezogene Vergütungsbestandteile oder vergleichbare, auf den besonderen Unternehmenserfolg bezogene wirtschaftliche Kenndaten. - In Betrieben, die sich in wirtschaftlichen Schwierigkeiten befinden, ist es möglich, die Anhebung der Entgelte um zwei Monate zu verschieben. Eine Verschiebung ist nur mit Zustimmung der Betriebsräte möglich.

Die vereinbarte tarifliche Abschlussrate bewirkt eine tabellenwirksame Anhebung der Tarifentgelte um 4,5\%. Aufgrund des Inkrafttretens zwischen dem 1.7. und 1.9. fällt die Tarifanhebung auf das ganze Jahr 2012 naturgemäß geringer aus. Unter Berücksichtigung der Basiseffekte des Abschlusses von 2011 ergibt sich nach unseren Berechnungen eine kalenderjährliche Erhöhung für 2012 von 2,9 \%. Diese liegt deutlich über der prognostizierten Steigerung der Verbraucherpreise für dieses Jahr um etwa $2 \%$.

\subsubsection{Weiterentwicklung des Tarifvertrages „Lebens-Arbeitszeit und Demografie“}

Der bereits 2008 geschlossene Vertrag (vgl. Bispinck/WSITarifarchiv 2009) wurde mit folgenden Vereinbarungen ergänzt bzw. modifiziert:

\section{Demografiefonds:}

- Die Arbeitgeber stellen den betrieblichen Demografiefonds von 2013 - 2015 zusätzlich jeweils $200 € / J a h r$ je TarifBeschäftigten zur Verfügung;

- eine Reduzierung auf $100 €$ aus wirtschaftlichen Gründen ist möglich;

- die Mittel können auf Basis einer freiwilligen Betriebsvereinbarung nur für einen oder mehrere der folgenden Zwecke verwendet werden: Langzeitkonten, Altersteilzeit und/ oder lebensphasenorientierte Arbeitszeitgestaltung.

Die Betriebsparteien können zur Ermöglichung der lebensphasenorientierten Arbeitszeitgestaltung ein sogenanntes Modell RV 80 (reduzierte Vollzeit mit 80 \% Arbeitszeit) vereinbaren. Mit dem Modell RV 80 können eine flexible Arbeitszeitgestaltung in bestimmten Lebensphasen und ein flexibler Übergang in den Ruhestand ermöglicht werden. Die Betriebsparteien legen durch freiwillige Betriebsverein- barung fest, welches Ziel im Betrieb mit der Einführung des Modells RV 80 verfolgt werden soll. Die Betriebsparteien können das Modell RV 80 auch beiden Zielen widmen.

Demografie-Korridor: Zur Bewältigung der demografischen Entwicklung kann im Einvernehmen zwischen Arbeitgeber und Betriebsrat befristet eine wöchentliche Arbeitszeit zwischen 35 und 40 Stunden vereinbart werden. Arbeitgeber und Arbeitnehmer können auf Grundlage einer freiwilligen Betriebsvereinbarung durch eine befristete individuelle Vereinbarung eine wöchentliche Arbeitszeit zwischen 35 und 40 Stunden festlegen (Wahlarbeitszeit). Ein Ausgleich für längere Arbeitszeiten erfolgt grundsätzlich in Zeit. Diese Korridor-Regelung ist zunächst auf drei Jahre befristet und kann nicht mit den bestehenden KorridorRegelungen für Arbeitszeit und Entgelt kombiniert werden.

\subsubsection{Perspektiven für Berufseinsteiger}

Um die Attraktivität der Perspektiven für Berufseinsteiger in der Chemie-Branche auszubauen, haben sich die Tarifvertragsparteien darauf verständigt, die einzelnen Bausteine für eine Karriere in der chemischen Industrie vor dem Hintergrund der demografischen Entwicklung weiterzuentwickeln und sie haben dazu einen Handlungskatalog vorgelegt.

Der Abschluss trägt in beiden Bestandteilen erkennbar Kompromisscharakter: Die im Vergleich zu anderen Abschlüssen hohe tarifliche Steigerungsrate konnte nur mit einer längeren Laufzeit erreicht werden. Wie sich die Bestimmungen zur Variabilisierung konkret auswirken werden, bleibt abzuwarten. Der materielle Wert des Abschlusses muss auch die zusätzlichen Arbeitgeberzahlungen in die Demografiefonds ab 2013 in Rechnung stellen. Die Regelungen zum Demografie-Tarifvertrag bringen verbesserte Möglichkeiten kollektiver und individueller Arbeitszeitgestaltung, aber sie schaffen zugleich den Unternehmen auch Raum für Arbeitszeitverlängerung.

Beide Tarifparteien werteten den Abschluss positiv: IG BCE-Verhandlungsführer Peter Hausmann sagte, es sei gelungen, den Beschäftigten „einen fairen Anteil“ an der wirtschaftlichen Entwicklung der Branche zu sichern. Der neue Demografietarifvertrag sei ein neuer tarifpolitischer Meilenstein auf dem Weg zu einer altersgerechten Arbeitswelt. Der BAVC sieht in dem Abschluss „einen fairen Kompromiss“. Die Kostenbelastung werde durch die lange Laufzeit, den Leermonat und den variablen Beginn der Tariferhöhung begrenzt. Durch die neuen Arbeitszeitregelungen würde es ermöglicht, künftig länger und flexibler zu arbeiten. Vor dem Hintergrund der alternden Gesellschaft sorgten die Tarifparteien gemeinsam für den „notwendigen Mentalitätswandel“ bei der Arbeitszeit.

\subsubsection{Branchenzuschläge}

Auch in der chemischen Industrie wurden Branchenzuschläge für Leiharbeitsbeschäftigte vereinbart. Bereits 
im Dezember 2011 hatten sich die Tarifparteien auf ein Grundsatzabkommen verständigt und Einvernehmen über Inhalt und Struktur eines Branchenzuschlagssystems hergestellt. Diese Regelungen wurden in einer Vereinbarung vom 19.6.2012 konkretisiert. Sie orientieren sich am Abschluss in der Metallindustrie. In fünf zeitlichen Stufen, die wie in der Metallindustrie gestaffelt sind, werden Zuschläge gezahlt: In den Entgeltgruppen 1 und 2 betragen die Branchenzuschläge in der 1. Stufe $15 \%$ - sie steigen bis auf $50 \%$ in der 5. Stufe. In den Entgeltgruppen 3 bis 5 starten die Zuschläge bei $10 \%$ und steigen bis auf $35 \%$ in der 5. Stufe. In der Endstufe erreichen die Leiharbeitnehmerinnen und Leiharbeitnehmer zwischen 85 und 90 \% der Chemie-Entgelte. Der Vertrag tritt zum 1.11.2012 in Kraft.

\section{Ausblick}

Im zweiten Halbjahr 2012 folgen Tarifverhandlungen in einer Reihe weiterer Branchen, darunter die Papier verarbeitende Industrie, regionale Bereiche des privaten Verkehrsgewerbes, der Kunststoffverarbeitung und der Energieversorgung, die Textil- und Bekleidungsindustrie sowie die Landwirtschaft. Die Ergebnisse dürften in etwa auf der Linie der bisherigen Abschlüsse liegen. Selbst bei niedrigeren Ergebnissen dürfte der Einfluss auf die gesamtwirtschaftliche Tarifentwicklung begrenzt sein, weil sie erst relativ spät im Laufe des Jahres in Kraft treten.

Die Tarifrunde 2013 wird nach den derzeit vorliegenden Kündigungsterminen zu Beginn bestimmt vom öffentlichen Dienst, wo die Tarifverträge für die Länder Ende 2012 auslaufen. Gleiches gilt für die Deutsche Bahn AG, die Holz und Kunststoff verarbeitende Industrie und die Wohnungswirtschaft. Größere Branchen folgen dann erst Ende März mit dem Bauhauptgewerbe sowie April und Mai mit dem Einzel- und Großhandel. Im April nächsten Jahres läuft auch der Metalltarifvertrag wieder aus.

\section{LITERATUR}

Bispinck, R. (2011): Welche finanziellen Wirkungen hat ein Tarifabschluss? Informationen zurTarifpolitik, Düsseldorf, http://www.boeckler.de/pdf/p_ta_ elemente_tarifabschluss_materieller_wert.pdf

Bispinck, R./WSI-Tarifarchiv (2009): Tarifpolitischer Jahresbericht 2008: Tarifpolitik in der Finanzmarktkrise, Düsseldorf, http://www.boeckler.de/pdf/ p_ta_jb_2008.pdf
Bispinck, R./WSI-Tarifarchiv (2011): Tarifpolitischer Jahresbericht 2010: Beschäftigungssicherung und gedämpfte Lohnentwicklung, in: WSI-Mitteilungen 64 (3), S. 123-130, http://www.boeckler.de/wsimit_2011_03_Bispinck.pdf Bispinck, R./WSI-Tarifarchiv (2012): Tarifpolitischer Jahresbericht 2011: Höhere Tarifabschlüsse - Konflikte um Tarifstandards - Neue Tarifregelungen, in: WSIInformationen zurTarifpolitik, Düsseldorf, http://www.boeckler.de/pdf/ p_ta_jb_2011.pdf

Burmeister, K. (2012): Leiharbeit - Tarif-Erfolg braucht Unterstützung durch linke Politik, in: Zeitschrift für sozialistische Politik und Wirtschaft (SPW) (190), S. 4

IG Metall (2012): Wirtschaftspolitische Informationen Nr. 01/01. März -Tarifrunde 2012 in der Metall- und Elektroindustrie. Sozialökonomische Rahmenbedingungen, http://www.igmetall-augsburg.de/downloads/artikel/attachments/ ARTID_50765_50Fsve?name=Infoblatt.pdf

Institut für Makroökonomie und Konjunkturforschung (IMK) (2012): Fiskalpakt belastet Euroraum, Gemeinsame Diagnose des Makro-Konsortiums IMK (Düs seldorf), OFCE (Paris) und WIFO (Wien), IMK-Report (71), März, Düsseldorf, http://www.boeckler.de/pdf/p_imk_report_71_2012.pdf

König, O./Detje, R. (2012): Fuß in derTür. Tarifabschluss Metall- und Elektroindustrie 2012: Junge Metaller gewinnen, in: Sozialismus 39 (365), S. 48-50 Riexinger, B. (2012): Zustimmung und Ablehnung. Zum Tarifergebnis im öffentlichen Dienst, in: Sozialismus 39 (364), S. 36-39

Towers-Watson (2012): Vorstandsvergütung im DAX 2011. Presseinformation vom 29.3. „DAX-Vorstandsbezüge mit Unternehmenserfolg um 12 Prozent gestiegen"

Vereinte Dienstleistungsgewerkschaft (ver.di) (2011): Tarifrunde 2012. TVöD Bund und Kommunen. Daten und Fakten, Berlin

Vereinte Dienstleistungsgewerkschaft (ver.di) (2012): Branchenzuschläge in der Leiharbeit - eine Alternative zu Equal-Pay? in: ver.di tarif.letter - Aktuelle Informationen zur ver.di-Tarifpolitik (03), S. 1-6

Vereinigung der kommunalen Arbeitgeberverbände (2012): Pressemappe der VkA zurTarifrunde 2012

Wendl, M. (2012): Der Abschluss im öffentlichen Dienst:Tarifpolitischer Erfolg, www.sozialismus.de 3.4.2012, http://www.sozialismus.de/nc/archiv/ kommentare_analysen/detail/artikel/tarifpolitischer-erfolg/

\section{AUTOREN}

REINHARD BISPINCK, Dr., ist Wissenschaftler im Wirtschafts- und Sozialwissenschaftlichen Institut (WSI) und Leiter des WSI-Tarifarchivs in der HansBöckler-Stiftung.

Reinhard-Bispinck@boeckler.de

GÖTZ BAUER, MONIKA MÜLLER, ULRICH SCHMIDT, MONIKA SCHWACKEPILGER, KATHRIN SONNEN, ANDREA TAUBE und MONIKA WIEBEL sind Sachbearbeiterinnen und Sachbearbeiter im WSI-Tarifarchiv. 